\title{
Gut Microbiota Dysbiosis Correlates with Abnormal Immune Response in Moderate COVID-19 Patients with Fever
}

\author{
Yaya Zhou',* \\ Xing Shi ${ }^{2,3, *}$ \\ Wei $\mathrm{Fu}^{\mathrm{l}, *}$ \\ Fei Xiang' \\ Xinliang $\mathrm{He}^{\prime}$ \\ Bohan Yang \\ Xiaorong Wang' \\ Wan-Li Ma' \\ 'Department of Respiratory and Critical \\ Care Medicine, Union Hospital, Tongji \\ Medical College, Huazhong University of \\ Science and Technology, Wuhan, 430022, \\ People's Republic of China; ${ }^{2}$ Department of \\ Respiratory and Critical Care Medicine, \\ Peking University People's Hospital, Beijing, \\ 100044, People's Republic of China; \\ ${ }^{3}$ Department of Pulmonary and Critical \\ Care Medicine, Shenzhen Institute of \\ Respiratory Diseases, First Affiliated \\ Hospital of Southern University of Science \\ and Technology, Shenzhen People's \\ Hospital, Shenzhen, 518020, People's \\ Republic of China \\ *These authors contributed equally to this \\ work
}

\begin{abstract}
Background: Most COVID-19 patients are moderate, and fever is the most common clinical manifestation and associated with poorer prognosis. Gut microbiota may also play important roles in COVID-19 pathogenesis. However, the association between gut microbiota and fever in individuals with moderate COVID-19 remains unclear.

Methods: We compared the clinical features and laboratory results of 187 moderate COVID-19 patients with fever and without fever and identified several inflammatory markers in patients with fever. Then, we performed gut metagenome-wide association study for 31 individuals to identify the microbes and their epitopes which have potential role in fever and hyperinflammation.
\end{abstract}

Results: Among 187 moderate COVID-19 patients, 127 (67.9\%) patients presented with fever. Lymphocytes, CD3 $+\mathrm{T}$ cells, CD4+ $\mathrm{T}$ cells and the ratio of CD4+ T cells to CD8+ T cells were significantly reduced, while AST, LDH, CRP, IL-6 and IL-10 were significantly elevated in patients with fever. Gut microbiome composition was significantly altered in patients with fever compared with those with non-fever. Opportunistic pathogens such as Enterococcus faecalis and Saccharomyces cerevisiae were enriched in patients with fever. E. faecalis was positively correlated with LDH and D-dimer and negatively correlated with CD8+T cells and IL-4, while $S$. cerevisiae was positively correlated with diarrhea symptom. Furthermore, several species with anti-inflammatory and protective effects, such as Bacteroides fragilis and Eubacterium ramulus, were enriched in patients with non-fever. B. fragilis was positively correlated with lymphocytes, and E. ramulus was negatively correlated with LDH, AST and IL-6. Finally, we found that several bacterial epitopes of GroEL, a homolog of human HSP60, were enriched in patients with fever and positively correlated with IL-6, IL-10, WBC, neutrophils, D-dimer, LDH, CRP, and E. faecalis.

Conclusion: Gut microbiota dysbiosis correlates with abnormal immune response in moderate COVID-19 patients with fever.

Keywords: moderate COVID-19, fever, shotgun metagenomic sequencing, gut microbiota, epitopes

\section{Introduction}

Wan-Li Ma

Department of Respiratory and Critical Care Medicine, Union Hospital, Tongji

Medical College, Huazhong University of

Science and Technology, Wuhan, 430022,

People's Republic of China

Tel +86-27-85726557

Fax +86-27-85726557

Email rong-100@I63.com;

whmawl@aliyun.com
Coronavirus disease 2019 (COVID-19) is an infectious illness caused by severe acute respiratory syndrome coronavirus 2 (SARS-CoV-2), with most COVID-19 cases being moderate. ${ }^{1}$ The most common clinical manifestation in moderate patients is fever, ${ }^{2}$ which is a hallmark of infection and inflammatory disease, and is commonly caused by the secretion of inflammatory mediators. The prevalence of fever was estimated to be $79.43 \%$ in symptomatic adult COVID-19 patients, ${ }^{3}$ which 
is less common than SARS $(99-100 \%),{ }^{4}$ but similar to middle east respiratory syndrome (MERS) (77\%, metaanalysis result). ${ }^{5}$ Moreover, several studies showed that the presence of fever was a predictor of adverse outcomes including intensive care unit (ICU) admissions and inhospital mortality, and every $0.5^{\circ} \mathrm{C}$ increase in body temperature had a significant increase in mortality. ${ }^{6,7}$ However, some patients only showed local symptoms such as sore throat ${ }^{8}$ and diarrhea. ${ }^{9-11}$ As the intestinal tract is the biggest immune organ, and its resident microflora is known to regulate host immune responses. ${ }^{12,13} \mathrm{We}$ speculate that the presence of fever may be related to the host immune status and inflammatory response mediated by gut microbiota.

Cytokine storms play a critical role in the pathogenesis of COVID-19, including acute respiratory distress syndrome (ARDS), multiple-organ dysfunction syndrome (MODS), and even death. ${ }^{14-17}$ The gut microbiota has a myriad of effects on the regulation of immune responses by promoting the production of pro-inflammatory cytokines including interleukin 6 (IL-6), tumor necrosis factor alpha (TNF- $\alpha$ ), interferon gamma (IFN- $\gamma$ ), and favoring the generation of regulatory $\mathrm{CD} 4+\mathrm{T}$ cells, and leading to the diversification of the B-cell repertoire and the production of $\mathrm{T}$ cell-dependent and -independent antibodies. ${ }^{12,13,18}$ IL-6 is one of the most important cytokines in the inflammatory storm in COVID-19 patients, ${ }^{15}$ meanwhile, it is also the most important mediator in the induction of fever. ${ }^{19}$ Several studies have suggested that the gut microbiota is associated with disease severity, and several cytokines and inflammatory markers in COVID-19 patients. $^{20,21}$ However, no study has elaborated on the association between gut microbiota and fever in patients with moderate COVID-19.

Here, we initially compared the clinical features and immune status of moderate COVID-19 patients with fever and without fever. We then utilized shotgun metagenomic sequencing to reveal the different profiles of gut microbiota in the two groups. Finally, we applied metagenomewide association study (MWAS) to identify gut microbes and microbial epitopes (MEs) that have a potential role in causing inflammation and fever.

\section{Materials and Methods}

\section{Participants and Sample Collection}

We recruited 187 patients ( $\geq 18$ years old) with confirmed moderate COVID-19 (127 patients with fever and 60 patients with non-fever) between January and March 2020. Patients with confirmed COVID-19 were defined as those who were positive for COVID-19 nucleic acid by real-time reverse transcriptase-polymerase chain reaction assays (RT-PCR) from throat-swab specimens. ${ }^{22}$ Moderate COVID-19 patients were defined as having fever and/or respiratory symptoms and/or other symptoms, and radiologic assessments found signs of pneumonia. ${ }^{22}$ Demographic information, comorbidities, date of symptom onset, symptoms and signs, laboratory test on admission, radiological images and treatment data of patients with COVID-19 were extracted from electronic medical records in Wuhan Union Hospital. Stool samples were collected within 72 hours on admission from 31 of the above 187 moderate patients with COVID-19 and immediately sent to the laboratory for inactivation at $56^{\circ} \mathrm{C}$ for 30 minutes, and then immediately sent to Wuhan Huada Gene Laboratory to detect fecal SARS-CoV-2 RNA and metagenomics. The study was approved by the Ethics Committee of Wuhan Union Hospital (2020-0149-02). All participants signed their written informed consent before participation, and this study was conducted in accordance with the Declaration of Helsinki. The fever group was defined as patients with an axillary temperature is higher than $37.3^{\circ} \mathrm{C}$, while the non-fever group was composed of COVID-19 patients with no fever $\left(\leq 37.3^{\circ} \mathrm{C}\right)$ during the course of the disease, including before admission and hospitalization in this study.

\section{Detection of SARS-CoV-2 RNA in Fecal Samples}

RNA was extracted from fecal samples and tested for SARS-CoV-2 via real-time RT-PCR using a previously described protocol. ${ }^{20}$

\section{DNA Extraction and Shotgun Metagenome Sequencing}

The microbial community DNA was extracted using MagPure Stool DNA KF kit B (Magen, China) following the manufacturer's instructions. DNA was quantified with a Qubit Fluorometer by using Qubit dsDNA BR Assay kit (Invitrogen, USA) and the quality was checked by running aliquot on $1 \%$ agarose gel. $1 \mu \mathrm{g}$ genomic DNA was randomly fragmented by Covaris. The selected fragments were treated through end repair, $3^{\prime}$ adenylated, adapters ligation, PCR amplifying and the products were purified to an average size of $200-400$ bp by the Magnetic beads. The double 
stranded PCR products were heat denatured and circularized by the splint oligo sequence. The single-strand circle DNA were formatted as the final library and qualified by QC. The qualified libraries were sequenced on MGI SEQ 2000 platform (BGI Shenzhen, china). All the raw sequences were trimmed by Trimmomatic v0.36 with the parameter SLIDINGWINDOW:4:20 and MINLEN:70. The trimmed reads were mapped to GRCh38 reference genome by Bowtie 2 to identify and remove host originated reads. The high-quality non-human reads were used to perform the subsequent analysis. The microbial taxonomic and MetaCyc pathway profiles were inferred using MetaPhlAn v2.7.6 and HUMAnA2, respectively. The taxonomic and functional profiles were normalized to relative abundance. Species occurring in more than $10 \%$ samples with a mean abundance larger than $10^{-5}$ were used to perform the differential abundance analysis and Spearman correlation analysis.

\section{Data Analysis}

The SPSS 25.0 statistical package for Windows was used for clinical data analysis. Demographic data and clinical characteristics were compared between the fever and nonfever groups. Median values with interquartile range (IQR) were used to describe continuous variables, and absolute or relative frequencies were used to describe categorical variables. We used the Mann-Whitney $U$-test to analyze continuous variables and the $\chi^{2}$ test or Fisher's exact test to analyze discrete variables in bivariate analyses. Level of significance was set at 0.05 (two-tailed).

Chaol diversity and Shannon index diversity were computed using the vegan package estimate $\mathrm{R}$ function and beta diversity was calculated using the Bray-Curtis distance. Based on the abundance profiles, the features (phyla, genera, and species) with significantly differential abundances across groups were determined using Wilcoxon's rank sum test, P-values $<0.05$ were considered significant. We applied linear discriminant analysis effect size (LEfSe) analysis to identify taxa differentially abundant between fever patients and nonfever patients. This method first uses the non-parametric factorial Kruskal-Wallis sum-rank test to detect features with significant differential abundance and then uses linear discriminant analysis (LDA) to calculate the effect size of each feature. To determine the correlation between gut microbiota taxonomies and clinical biomarkers or MEs, the Spearman rank correlation coefficient was calculated using cor.test functions in R. Differentially enriched Kyoto Encyclopedia of Genes and Genomes (KEGG) pathways were identified based on reporter scores. An absolute value of reporter score of 1.65 or higher was used as the detection threshold for significance.

Epitope reference sequences were downloaded from the Immune Epitope Database (IEDB), and the all highquality reads were aligned to the reference sequence using BLAST with E-value $10^{-5}$, and only the best hit was retained. The abundance of each epitope in a sample was calculated by summing all the reads aligned to it, and then divided by total reads $/ 1,000,000$.

\section{Results}

\section{Demographic and Clinical Characteristics of the Participants}

A total of 187 moderate COVID-19 patients (122 women and 65 men) were included in this study with an average age of 39 (IQR, 32-57) years. Among them, 60 (32.1\%) patients did not present with fever while 127 (67.9\%) patients presented with fever. Compared with non-fever patients, patients with fever had a lower proportion of sputum symptom, a higher proportion of fatigue symptom and a shorter time from illness onset to first admission. Among patients with fever, lymphocytes, CD3 $+\mathrm{T}$ cells, CD4+ T cells and the ratio of CD4+ T cells to CD8+ T cells were significantly lower while aspartate aminotransferase (AST), lactate dehydrogenase (LDH), C-reactive protein (CRP), IL-6 and interleukin 10 (IL-10) were significantly higher. The demographic and clinical characteristics, as well as laboratory results on admission of 127 patients with fever and 60 patients without fever are shown in Tables 1 and 2 .

Fecal samples of 31 individuals (20 patients with fever and 11 patients without fever) from the above 187 moderate patients were subjected to metagenomic sequencing and detection of fecal SARS-CoV-2 RNA (1 stool sample per patient). There were 4 patients with SARS-CoV-2 positive faeces and 27 patients with SARS-CoV-2 negative faeces; moreover, 4 patients with SARS-CoV-2 positive faeces were all in fever group. There were no significant differences between the two groups based on sex, age, symptoms, comorbidities, etc. The demographic and clinical characteristics, as well as laboratory results on admission of 31 patients are shown in Table S1 and Table S2.

\section{Taxonomic Differences in Gut Microbiota Between Moderate COVID-19 Patients with Fever and without Fever}

To investigate the composition and function of the gut microbiota in moderate COVID-19 patients, we performed 
Table I Demographic and Clinical Characteristics of 187 Moderate Patients with COVID-19 on Admission

\begin{tabular}{|c|c|c|c|c|}
\hline Characteristics & $\begin{array}{l}\text { All Patients } \\
\qquad(n=187)\end{array}$ & $\begin{array}{l}\text { Patients without Fever } \\
\qquad(n=60)\end{array}$ & $\begin{array}{l}\text { Patients with Fever } \\
\qquad(n=127)\end{array}$ & $P$ value \\
\hline Age, years & $39.0(32.0-57.0)$ & $48.0(32.0-64.8)$ & $37.0(32.0-53.0)$ & 0.091 \\
\hline $\begin{array}{l}\text { Gender } \\
\text { Male } \\
\text { Female }\end{array}$ & $\begin{array}{l}65(34.8) \\
122(65.2)\end{array}$ & $\begin{array}{l}19(31.7) \\
41(68.3)\end{array}$ & $\begin{array}{l}46(36.2) \\
81(63.8)\end{array}$ & 0.542 \\
\hline \multicolumn{5}{|l|}{ Symptoms } \\
\hline Cough & $116(62.0)$ & $36(60.0)$ & $80(63.0)$ & 0.694 \\
\hline Sputum & $54(28.9)$ & $24(40.0)$ & $30(23.6)$ & 0.021 \\
\hline Myalgia & $47(25.1)$ & II (I8.3) & $36(28.3)$ & 0.141 \\
\hline Dyspnea & 78 (4I.7) & $20(33.3)$ & $58(45.7)$ & 0.110 \\
\hline Fatigue & $85(45.5)$ & $21(35.0)$ & $64(50.4)$ & 0.048 \\
\hline Pharyngalgia & $33(17.6)$ & $8(13.3)$ & $25(19.7)$ & 0.288 \\
\hline Digestive Symptoms & $57(30.5)$ & $15(25.0)$ & $42(33.1)$ & 0.263 \\
\hline Nausea & $8(4.3)$ & $4(6.7)$ & $4(3.1)$ & 0.470 \\
\hline Vomiting & $13(7.0)$ & $5(8.3)$ & $8(6.3)$ & 0.839 \\
\hline Diarrhea & $50(26.7)$ & $12(20.0)$ & $38(29.9)$ & 0.152 \\
\hline Highest temperature, ${ }^{\circ} \mathrm{C}$ & & & & $<0.001$ \\
\hline$\geq 39.0$ & $32(17.1)$ & $0(0.0)$ & $32(25.2)$ & \\
\hline$<39.0$ & $155(82.9)$ & $60(100.0)$ & $95(74.8)$ & \\
\hline Length of fever, days & $8.0(3.0-11.0)$ & NA & $8.0(3.0-11.0)$ & NA \\
\hline Comorbidities & $50(26.7)$ & $20(33.3)$ & $30(23.6)$ & $0.16 \mathrm{I}$ \\
\hline Hypertension & $30(16.0)$ & II (I8.3) & $19(15.0)$ & 0.557 \\
\hline Cardiovascular disease & $4(2.1)$ & I (I.7) & $3(2.4)$ & 1.000 \\
\hline Diabetes & II (5.9) & $3(5.0)$ & $8(6.3)$ & 0.984 \\
\hline Chronic lung disease & $7(3.7)$ & $3(5.0)$ & $4(3.1)$ & 0.834 \\
\hline Chronic kidney disease & $2(1.1)$ & I (I.7) & $\mathrm{I}(0.8)$ & 1.000 \\
\hline Nervous system disease & $4(2.1)$ & $2(3.3)$ & $2(1.6)$ & 0.815 \\
\hline Digestive system disease & $5(2.7)$ & $2(3.3)$ & $3(2.4)$ & 1.000 \\
\hline Hypothyroidism & $6(3.2)$ & $2(3.3)$ & $4(3.1)$ & 1.000 \\
\hline $\begin{array}{l}\text { Supplemental oxygen required } \\
\text { Nasal cannula }\end{array}$ & $100(53.5)$ & $30(50.0)$ & $70(55.1)$ & 0.512 \\
\hline $\begin{array}{l}\text { Onset of symptoms to first medical visitation, } \\
\text { days }\end{array}$ & $2.0(1.0-5.0)$ & $2.0(0.3-6.8)$ & $2.0(1.0-5.0)$ & 0.729 \\
\hline Onset of symptoms to first admission, days & $9.0(5.0-15.0)$ & $12.5(6.0-20.8)$ & $7.0(5.0-12.0)$ & 0.004 \\
\hline Length of hospital, days & $17.0(11.0-25.0)$ & $16.5(10.0-27.0)$ & $18.0(12.0-22.0)$ & 0.499 \\
\hline Discharged & $187(100.0)$ & $60(100.0)$ & $127(100.0)$ & NA \\
\hline
\end{tabular}

Notes: Digestive Symptoms included nausea, vomiting, and diarrhea. Data are median (IQR) or $\mathrm{n}(\%)$. $p$ values were calculated by Mann-Whitney U-test, $\chi^{2}$ test, or Fisher's exact test, as appropriate. $P$ value $<0.05$ is considered to be statistically significant.

metagenomic sequencing of stool samples from 31 patients (their clinical information and sequencing statistics are detailed in Table S3 and Table S4). The alpha diversity measured using two different methods was lower in patients with fever than in patients without fever. However, these differences were not statistically significant based on Wilcoxon rank sum test (Chao1, $\mathrm{p}=0.055$; Shannon, $\mathrm{p}=0.3$ ) (Figure $1 \mathrm{~A}$ ). The Bray-Curtis distance showed that the gut microbiota composition differed between the two groups $(\mathrm{p}<0.001)$ (Figure 1A). We then compared gut microbiota composition between the patients with fever and without fever. At the phylum 
Table 2 Laboratory Results of 187 Moderate Patients with COVID-19 on Admission

\begin{tabular}{|c|c|c|c|c|}
\hline & All Patients $(n=187)$ & Patients without Fever $(n=60)$ & Patients with Fever $(n=127)$ & $P$ value \\
\hline \multicolumn{5}{|l|}{ Hematologic } \\
\hline WBC, $109 / \mathrm{mL}$ & $4.7(3.8-6.1)$ & $5.1(4.1-6.1)$ & $4.5(3.6-6.1)$ & 0.073 \\
\hline Neutrophils, $10^{9} / \mathrm{mL}$ & $3.0(2.1-3.9)$ & $3.2(2.4-3.8)$ & $2.9(1.9-4.1)$ & 0.297 \\
\hline Lymphocytes, $10^{9} / \mathrm{mL}$ & $1.2(0.9-1.6)$ & $1.4(1.0-1.7)$ & $1.2(0.9-1.6)$ & 0.036 \\
\hline \multicolumn{5}{|l|}{ T lymphocytes } \\
\hline $\mathrm{CD} 3+(\%)$ & $74.7(70.3-79.9)$ & $76.4(74.5-82.4)$ & $74.3(69.0-79.5)$ & 0.011 \\
\hline CD4+ (\%) & $43.3(37.8-48.5)$ & $44 . I(42.5-5 \mid .4)$ & $42.6(36.7-45.8)$ & $<0.001$ \\
\hline CD8+ (\%) & $26.1(21.5-29.6)$ & $26.1(20.7-30.7)$ & $26.0(22.1-29.2)$ & 0.860 \\
\hline CD4+/CD8+, Ratio & $1.8(1.3-2.1)$ & $1.9(1.4-2.4)$ & $1.7(1.3-1.9)$ & 0.017 \\
\hline \multicolumn{5}{|l|}{ Biochemical } \\
\hline $\mathrm{ALT}, \mathrm{U} / \mathrm{L}$ & $19.0(15.0-29.7)$ & $18.5(13.3-23.8)$ & $20.0(15.0-33.0)$ & 0.184 \\
\hline AST, U/L & $22.0(17.0-29.0)$ & $20.5(16.3-27.0)$ & $23.0(|8.0-3| .0)$ & 0.035 \\
\hline $\mathrm{LDH}, \mathrm{U} / \mathrm{L}$ & $197.0(167.0-242.0)$ & $183.0(152.3-229.0)$ & $204.0(175.0-247.0)$ & 0.003 \\
\hline CK, U/L & $58.0(44.0-92.0)$ & $56.0(45.0-83.0)$ & $60.0(44.0-96.0)$ & 0.521 \\
\hline Urea nitrogen, $\mathrm{mmol} / \mathrm{L}$ & $4.0(3.1-4.8)$ & $4.1(3.3-5.1)$ & $3.8(3.0-4.5)$ & 0.058 \\
\hline Creatinine, $\mu \mathrm{mol} / \mathrm{L}$ & $67.0(58.5-78.1)$ & $67.5(58.6-74.5)$ & $66.9(58.5-79.2)$ & 0.749 \\
\hline \multicolumn{5}{|l|}{ Infection-related markers } \\
\hline CRP, mg/L & $6.6(3.1-15.3)$ & $3.1(3.1-6.6)$ & $9.9(3.1-20.2)$ & $<0.001$ \\
\hline PCT, $\mu g / L$ & $0.1(0.1-0.1)$ & $0.1(0.1-0.1)$ & $0.1(0.1-0.1)$ & $0.64 I$ \\
\hline $\mathrm{IL}-2, \mathrm{pg} / \mathrm{mL}$ & $2.7(2.4-2.8)$ & $2.7(2.5-2.8)$ & $2.6(2.4-2.8)$ & 0.315 \\
\hline $\mathrm{IL}-4, \mathrm{pg} / \mathrm{mL}$ & $2.0(1.7-2.3)$ & $2.0(1.9-2.3)$ & $2.0(1.6-2.3)$ & 0.090 \\
\hline IL-6, pg/mL & $5.8(3.4-8.8)$ & $5.0(3.0-8.6)$ & $5.9(3.7-11.8)$ & 0.037 \\
\hline $\mathrm{IL}-10, \mathrm{pg} / \mathrm{mL}$ & $4.0(3.2-4.6)$ & $3.9(3.0-4.2)$ & $4.0(3.3-5.0)$ & 0.015 \\
\hline TNF- $\alpha, p g / m L$ & $2.2(1.9-2.4)$ & $2.3(2.1-2.4)$ & $2.2(1.9-2.5)$ & 0.073 \\
\hline IFN- $\gamma, \mathrm{pg} / \mathrm{mL}$ & $2.2(1.9-2.5)$ & $2.2(1.9-2.3)$ & $2.2(1.9-2.6)$ & 0.508 \\
\hline D-dimer, $\mu g / L$ & $0.3(0.2-0.6)$ & $0.4(0.2-0.7)$ & $0.3(0.2-0.6)$ & 0.946 \\
\hline
\end{tabular}

Notes: Data are median (IQR). $P$ values were calculated by Mann-Whitney U-test. $P$ value $<0.05$ is considered to be statistically significant.

Abbreviations: WBC, white blood cell; ALT, alanine aminotransferase; AST, aspartate aminotransferase; LDH, lactate dehydrogenase; CK, creatine Kinase; CRP, C-reactive protein; PCT, procalcitonin; IL-2, interleukin-2; IL-4, interleukin-4; IL-6, interleukin-6; IL- 10, interleukin- I0; TNF- $\alpha$, tumor necrosis factor- $\alpha$; IFN- $\gamma$ : interferon- $\gamma$.

level, the relative abundance of Bacteroidetes was much lower while that of Ascomycota was significantly higher in the fever group (Table S5). At the genus level, the relative abundance of gut microbiota also differed between the two groups (Figure 1B and Table S6), and results of LEfSe (minimum LDA score: 2.0) showed that Saccharomyces and Enterococcus were significantly enriched in patients with fever, whereas Anaerostipes, Prevotella, Parabacteroides, Phascolarctobacterium, Eggerthella were enriched in patients with non-fever (Figure 2). At the species level, the heatmap analysis further showed the composition of the gut microbiota differed between patients with fever and without fever (Table S7 and Figure S1). These results indicate that opportunistic pathogens were enriched in patients with fever while butyrateproducing bacteria Anaerostipes, were enriched in patients with non-fever.

\section{Correlation Between Clinical Indices and Gut Microbiota}

We found the significantly different species in the two groups, with mean relative abundance $>10^{-5}$ (Figure 3A). Five kinds of species were significantly enriched in patients with fever, namely Enterococcus faecalis, Citrobacter freundii, Citrobacter unclassified, Haemophilus parainfluenzae, and Saccharomyces cerevisiae, and six species were significantly enriched in patients with non-fever, namely Bacteroides cellulosilyticus, Bacteroides fragilis, Bacteroides thetaiotaomicron, Bacteroides xylanisolvens, Eubacterium ramulus, and Erysipelotrichaceae bacterium_6_1_45 (Figure 3A). This result shows that patients with fever have significantly more pathogens but lack butyrate-producing species, which may lead to local overactivated immunity 
A
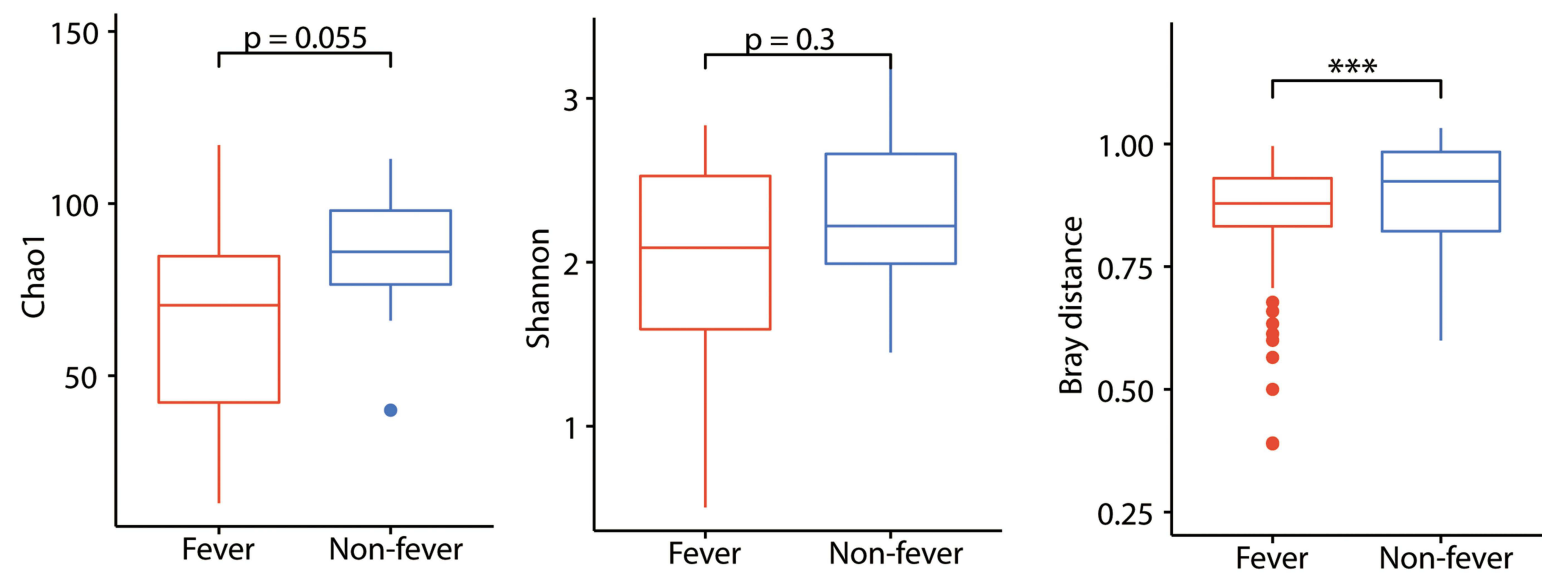

B

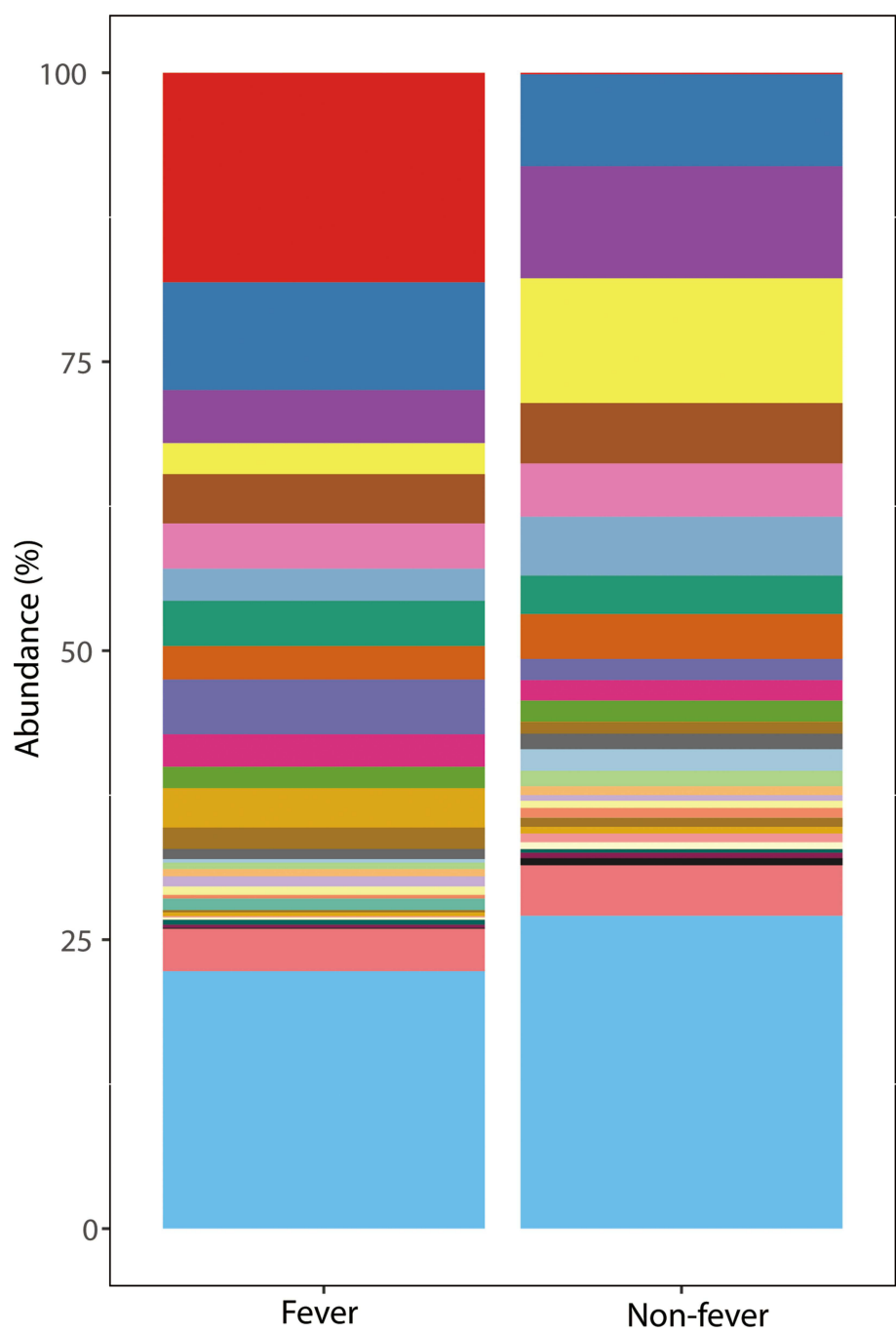

Genus

\begin{tabular}{|l|l|}
\hline Enterococcus & Anaerostipes \\
\hline Blautia & Rose buria \\
\hline Esche richia & Subdolig ranulum \\
\hline Bacteroides & Coprococcus \\
\hline Megamonas & Dialister \\
\hline Eubacte rium & Citrobacter \\
\hline Akkermansia & Erysipelatoclost ridium \\
\hline Ruminococcus & Peptoclostridium \\
\hline Streptococcus & Prevotella \\
\hline Bifidobacte rium & Phascolarctobacte rium \\
\hline Clost ridium & Faecalibacte rium \\
\hline Lachnoclost ridium & Collinsella \\
\hline Klebsiella & Parabacteroides \\
\hline Veillonella & Others \\
\hline Dorea & Unclassfied \\
\hline
\end{tabular}

Coprobacillus

Figure I Results of diversity and taxonomy. (A) gut microbiota richness (Chao I) and gut microbiota diversity (Shannon index) are not significantly different in the gut microbiota of patients with fever and without fever, and the beta diversity (Bray distance) shows the gut microbiota composition is different between patients with fever and without fever. (B) The bar plots at genus level shows differences in relative abundance of gut microbiota between two groups, $* * * p<0.001$ by the Wilcoxon rank-sum test. 


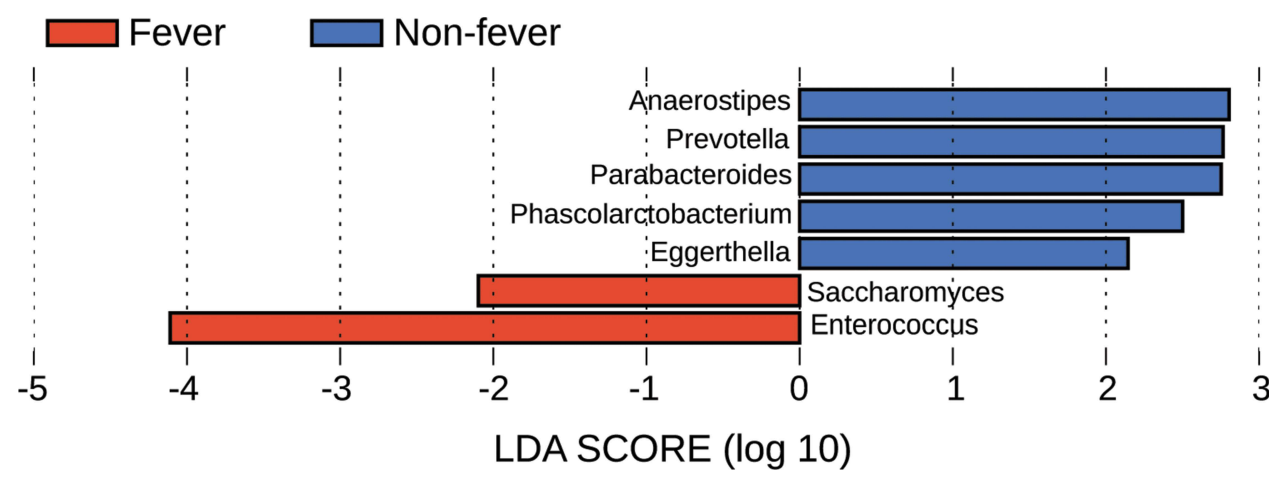

Figure 2 Linear discrimination analysis (LDA) effect size (LEfSe) results comparing patients with fever and without fever. Histogram of the LDA scores computed for differentially abundant genera between the two groups. The LDA scores $(\log 10)>2$ are list.

and linked to the hyperinflammation in fever. Spearman's rank correlation test also showed that the significantly enriched species in patients with fever were associated with the clinical indices. For example, $S$. cerevisiae was positively correlated with diarrhea (rho $=0.55, \mathrm{p}=0.001$ ), and E. faecalis was positively correlated with $\mathrm{LDH}(\mathrm{rho}=0.54, \mathrm{p}=0.002)$ and $\mathrm{D}$-dimer (rho=0.55, $\mathrm{p}=0.001$ ), and negatively correlated with $\mathrm{CD} 8+\mathrm{T}$ cells $(\mathrm{rho}=-0.42, \mathrm{p}=0.02)$ and interleukin 4 (IL4) $(\mathrm{rho}=-0.42, \mathrm{p}=0.02)$ (Figure $3 \mathrm{~B})$. Among the significantly enriched species in patients with non-fever, E. bacterium_6_1_45 and B. fragilis were positively correlated with lymphocytes $(\mathrm{rho}=0.50, \mathrm{p}=0.004$; rho $=0.37, \mathrm{p}=0.04$, respectively), and E. ramulus was negatively correlated with $\mathrm{LDH}(\mathrm{rho}=-0.42, \mathrm{p}=0.02)$, $\operatorname{AST}(\mathrm{rho}=-0.38, \mathrm{p}=0.03)$ and IL-6 (rho $=-0.44, \mathrm{p}=0.01)$ (Figure $3 \mathrm{~B}$ ). In conclusion, these results suggest that the clinical indices are closely associated with the imbalance of the intestinal microbiota.

\section{Gut Microbiota in Patients with Fever Have Significantly More GroEL Epitopes}

We predicted the MEs compositions in the shotgun metagenomic sequences to discover the potential MEs that correlate with the hyperinflammation in patients with fever. To identify specific ME markers that distinguish patients with fever from those without fever, we performed differential abundance analysis and found 27 differential MEs (Figure 4A and Table S8). Interestingly, five epitopes derived from GroEL were enriched in the fever group, ie EVASKTADKAGDGTTTATVLAEAIYTEGLR, IAKLE NPYILITDKKVSSIKDILPILEEIM， LPTLVLNKIRGTF NVVAVKAPGFGDRRKAM，DPVKVTRIALQNAVSVS SMLLTTEAVIYDV, and VASKTADEAGDGTTTATV
LAYGVFKEGLRN. Moreover, some of these five epitopes were positively correlated with IL-6, IL-10, white blood cell (WBC), neutrophils, CRP, D-dimer, and LDH (Figure 4B). The strong positive correlation between GroEL epitopes and clinical indices shows that the crossactivity of gut microbiota and host may play an important role in aggravating inflammatory reactions in patients with fever. In addition, four of the five epitopes were all positively correlated with E. faecalis (Figure 4C), whose relative abundance was significantly higher in patients with fever.

\section{Functional Alteration in Gut Microbiota in COVID-19 Patients with and without Fever}

We explored the functional features of the gut microbiota across the two groups by annotating the gene catalog based on the KEGG pathway. Pathways differing between patients with fever and without fever with an absolute value of reporter score $>1.65$ were identified. Among two groups, 95 KEGG pathways were significantly different in the two groups. Fifty-six KEGG pathways were enriched in patients with fever (Table S9), and 43 of the 56 KEGG pathways belong to metabolism, mainly including carbohydrate metabolism, amino acid metabolism, energy metabolism, metabolism of cofactors and vitamins, and glycan biosynthesis and metabolism. Thirty-nine KEGG pathways were enriched in patients with nonfever (Table S10), 18 of the 39 KEGG pathways belong to genetic information processing, mainly including replication and repair, translation, transcription, and folding, sorting and degradation; 9 of the 39 KEGG pathways belong to metabolism, mainly including glycan biosynthesis and metabolism and lipid metabolism. 
A

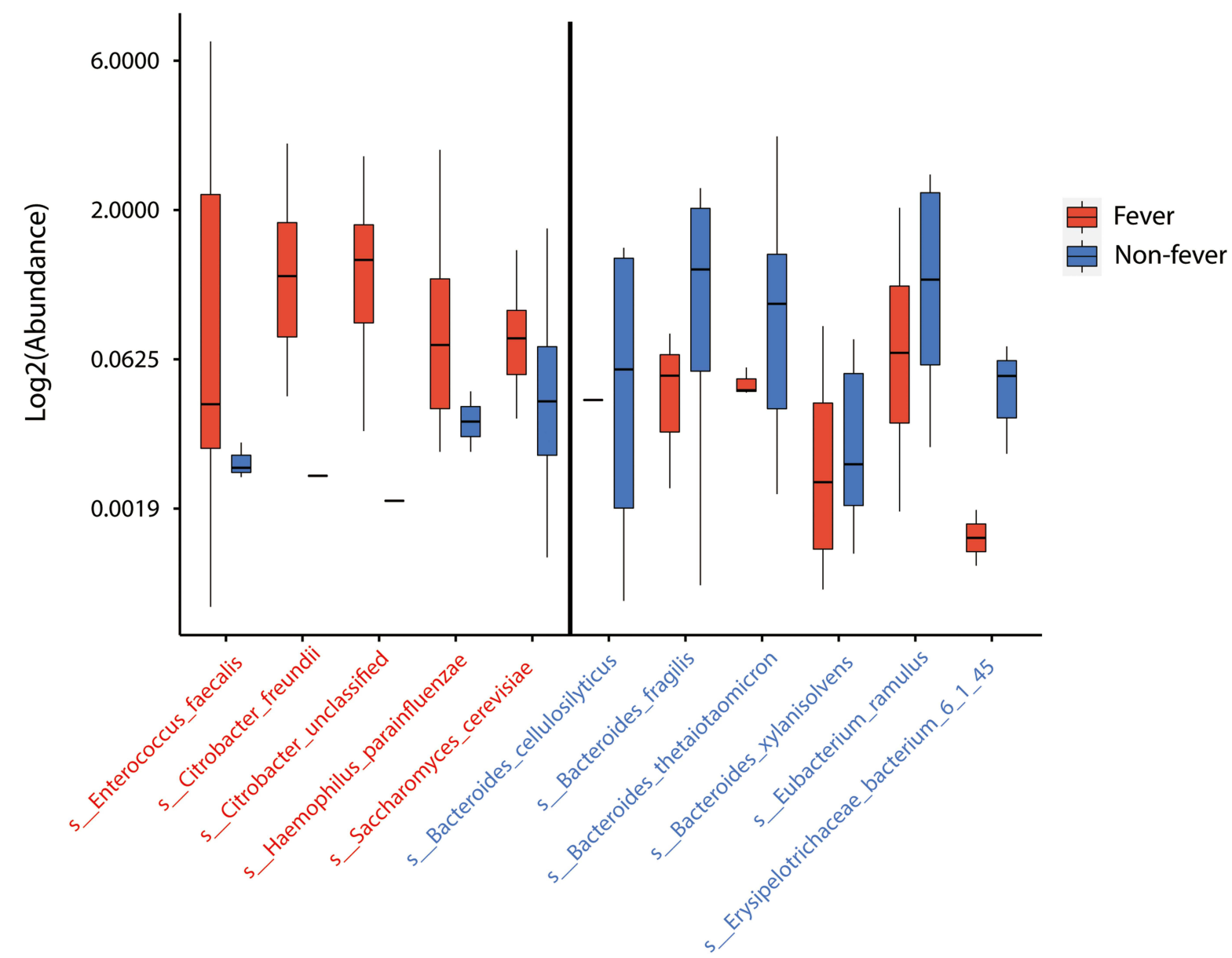

B

s_Citrobacter_unclassified

s_Citrobacter_freundii

s_Saccharomyces_cerevisiae

s_Enterococcus_faecalis

s_Bacteroides_thetaiotaomicron

s_Bacteroides_cellulosilyticus

s_Haemophilus_parainfluenzae

s_Erysipelotrichaceae_bacterium_6_1_45

s_Eubacterium_ramulus

S__Bacteroides_xylanisolvens

s_Bacteroides_fragilis
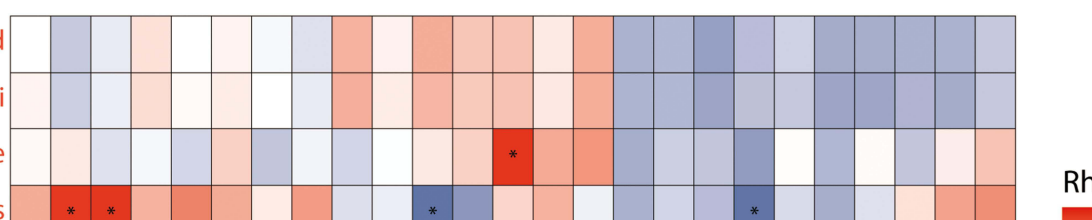

Rho

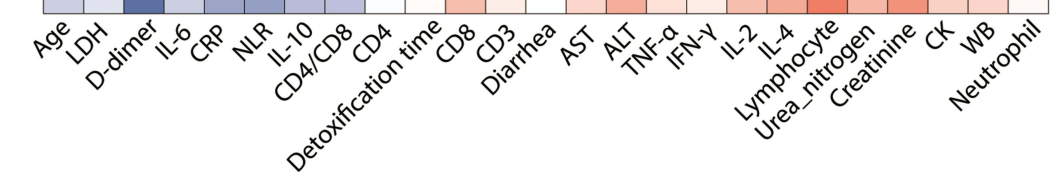

Figure 3 Altered gut microbiota composition associated with clinical indices in patients with moderate COVID-19. (A) differential species with mean relative abundance $>$ $10^{-5}$ in patients with and without fever are showed. Species that are enriched in patients with and without fever are marked in red and blue, respectively. The relative abundance is shown using boxplots. Boxes represent the inter quartile ranges, lines inside the boxes denote medians. (B) differential species are correlated with clinical indices in COVID-19 patients. Spearman's rank correlation coefficient is indicated using a color gradient: red indicates positive correlation; purple indicates negative correlation, ${ }^{*} \mathrm{p}<0.05$. 
A
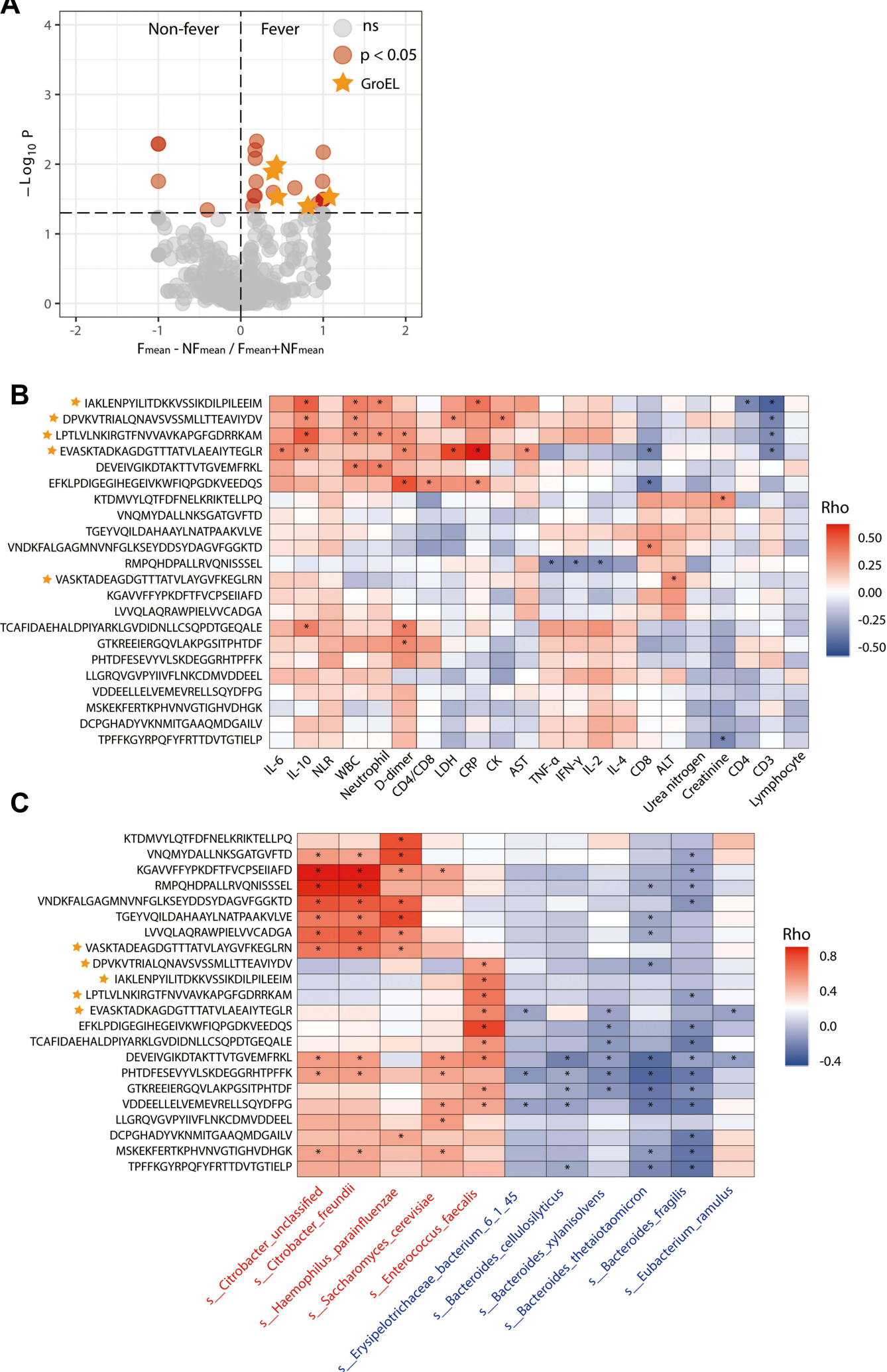

Figure 4 Gut microbial epitopes (MEs) in patients with moderate COVID-19. (A) different epitopes from gut microbiota in moderate COVID-I9 patients with and without fever are shown. (B) MEs enriched in fever group are correlated with clinical indices. (C) MEs enriched in fever group are correlated with different gut microbiota species. Spearman's rank correlation coefficient is indicated using a color gradient: red indicates positive correlation; purple indicates negative correlation, ${ }^{*}<0.05$. Species that are enriched in patients with and without fever are marked in red and blue, respectively. 


\section{Discussion}

The gut microbiota plays an essential role in the progression of COVID-19 via the regulation of immune responses. Zuo et al and Yeoh et al found that the gut microbiota composition was altered in COVID-19 patients and was associated with SARSCoV-2 levels in fecal samples, disease severity, plasma concentrations of several cytokines and inflammatory markers. ${ }^{20,21}$ However, our study is the first to investigate the role of gut microbiota and their epitopes in moderate COVID19 patients with fever and without fever, using metagenomewide association method. To the best of our knowledge, this is also the first clinical study to explore the gut microbiota of COVID-19 patients from Wuhan city. Our research identified differences in clinical features and inflammatory responses, as well as specific signatures of the fecal microbiota between moderate COVID-19 patients with fever and without fever.

Studies have demonstrated that gut microbiota plays a certain role in the pathogenesis of febrile diseases, such as familial Mediterranean fever ${ }^{23}$ and typhoid fever. ${ }^{24}$ However, whether gut microbiota affects the presence of fever in COVID-19 patients is currently unknown. Through MWAS, we found that the abundance of E. faecalis, C. freundii, C. unclassified, H. parainfluenzae, and S. cerevisiae in the guts of COVID-19 patients with fever were significantly higher than in COVID-19 patients with non-fever. In addition, E. faecalis was positively associated with $\mathrm{LDH}$ and D-dimer, and $S$. cerevisiae was positively correlated with diarrhea. It is well documented that $E$. faecalis is responsible for a serious healthcare problem and most patients infected with this pathogen suffer from severe inflammation and tissue injury. ${ }^{25,26}$ Saccharomyces was found to be the most abundant fungal genus in human stool, followed by Malassezia and Candida. ${ }^{27}$ Diarrhea may be associated with reduction in normal flora or a toxic effect of $S$. cerevisiae on the intestinal wall. $^{28}$ Studies found C. freundii, C. unclassified, and $H$. parainfluenzae belong to opportunistic pathogens that cause various human infections. ${ }^{29-31}$ B. cellulosilyticus, B. fragilis, B. thetaiotaomicron, B. xylanisolvens, E. ramulus, and E. bacterium_6_1_45 were significantly enriched in COVID-19 patients with non-fever. Multiple studies have shown that these enriched species in patients with non-fever can prevent pathogen invasion by enhancing mucosal barrier functionality within the gut, increasing short-chain fatty acids production, promoting the preferential differentiation of antiinflammatory Treg/Th2 cells, and suppressing the relative differentiation of pro-inflammatory Th1/Th17 cells, thereby reducing inflammatory cytokine production. ${ }^{32-36}$
It is worth noting that we found that five MEs (GroEL) enriched in patients with fever were positively correlated with IL-6, IL-10, WBC, neutrophils, and CRP, as well as with bacteria $E$. faecalis enriched in patients with fever. Bacterial GroEL is a homolog of human heat shock protein 60 (HSP60), and can induce various cross-immune reactions in the human body. ${ }^{37,38}$ The link between HSP and fever is inseparable. Kol et $\mathrm{al}^{39}$ showed that HSP60 can stimulate TNF- $\alpha$ secretion by macrophages and can also stimulate endothelial cells, smooth muscle cells and macrophages to produce adhesion factors and pro-inflammatory cytokines including interleukin 11 (IL-11), interleukin 8 (IL-8), interleukin 12 (IL-12), and IL-6, which is the most important mediator for the induction of fever. ${ }^{19}$ The cytokine IL-6 is released upon peripheral immune challenge, and stimulates the production of pyrogenic substances, which are critical in the febrile response. ${ }^{40}$ On the other hand, it may also be directly associated with different immune responses in patients with and without fever. Based on the above results, we believe that the gut microbiota plays a role in the induction and progression of fever in COVID-19 patients, and hypothesize that fever in COVID-19 patients may be partly caused by increased opportunistic pathogens in the gut, which can stimulate our bodies to secrete inflammatory cytokines such as IL-6. Conversely, the absence of fever in COVID-19 patients may be due to the higher content of anti-inflammatory bacteria in the gut. And the further mechanism may be that Enterococcus faecalis GroEL affects the induction of fever by stimulating the production of IL-6. However, this hypothesis needs to be verified.

Previous studies have shown that fever is a predictor of poor prognosis in COVID-19 patients. ${ }^{6,7,41}$ In our study, the patients included are all moderate patients, and there was no significant difference in the survival rate and hospital length of stay of patients with fever and without fever, which may be due to the limitation of sample size and the composition of different regions and populations. However, we subsequently followed up 55 of the above 187 patients at three months after discharge, and found that COVID-19 patients with fever in the acute phase had a higher proportion of persistent symptoms at three months after discharge, especially myalgia, which was significant difference in the two groups $(54.5 \%$ vs $9.1 \%, \mathrm{p}=0.018)$ (unpublished data). These findings suggest that patients with fever in the acute phase are more likely to have persistent symptoms at later stages, which may be associated with altered gut microbiota. As far as fever, immune response and gut microbiota are concerned, their causality and interaction mechanisms need to be studied further. 
Our study had several limitations. First, we only conducted the metagenomics study of stool samples. If combined with metabolomics, transcriptomics and proteomics, we can further increase our understanding of the mechanism of fever in moderate COVID-19 patients. Second, lifestyle and diet may further affect gut microbiota composition. We did not have information on lifestyle, but all patients had a similar diet during hospitalization because their meals were provided by the restaurant of Wuhan Union Hospital. Third, stool samples were collected after all patients received antibiotic treatment, which may have affected the intestinal flora, fortunately, all patients used the same antibiotics (moxifloxacin). In other words, the species comparison between COVID-19 patients with fever and without fever was performed under the same background. Fourth, we collected only one stool specimen from each patient within 72 hours on admission and did not collect stool specimens at different time points during the course of the disease. We are currently analyzing the gut microbiota of patients after discharge to study the dynamic changes of gut microbiota and the long-term impact on COVID-19 patients.

\section{Conclusions}

Our study found that several opportunistic pathogens were significantly enriched in patients with fever, while beneficial bacteria that have anti-inflammatory effects and protect the intestinal mucosal barrier were significantly enriched in patients with non-fever. Gut MEs of GroEL are enriched in patients with fever and have strong correlations with inflammation markers, indicating their potential role in simulating the production of inflammatory mediators and causing fever. Our study, together with previous studies, suggests that gut microbiota dysbiosis correlates with abnormal immune response in moderate COVID-19 patients with fever and gut microbiota may play an important role in immune status and inflammatory response of COVID-19 patients.

\section{Abbreviations}

COVID-19, Coronavirus Disease 2019; SARS-CoV-2, severe acute respiratory syndrome coronavirus 2 ; MERS, middle east respiratory syndrome; ICU, intensive care unit; ARDS, acute respiratory distress syndrome; MODS, multiple-organ dysfunction syndrome; IL-6, interleukin 6 ; TNF- $\alpha$, tumor necrosis factor alpha; IFN-r, interferon gamma; MWAS, metagenomewide association study; MEs, microbial epitopes; RT-PCR, real-time reverse transcriptase-polymerase chain reaction assays; IQR, interquartile range; LEfSe, linear discriminant analysis effect size; LDA, linear discriminant analysis;
KEGG, Kyoto Encyclopedia of Genes and Genomes; IEDB, Immune Epitope Database; AST, aspartate aminotransferase; $\mathrm{LDH}$, lactate dehydrogenase; CRP, C-reactive protein; IL-10, interleukin 10; IL-4, interleukin 4; WBC, white blood cell; HSP60, heat shock protein 60 ; IL-11, interleukin 11; IL-8, interleukin 8; IL-12, interleukin 12.

\section{Data Sharing Statement}

All data generated or analysed during this study are included in this published article and its supplementary file.

\section{Ethics Approval and Informed Consent}

The study was approved by the Ethics Committee of Wuhan Union Hospital (2020-0149-02). All participants signed their written informed consent before participation, and this study was conducted in accordance with the Declaration of Helsinki.

\section{Acknowledgments}

This study gratefully acknowledges the patients who participated in the research.

\section{Funding}

This work was supported by the Fundamental Research Funds for the Central Universities (No. 2020kfyXGYJ034) and (No. 2020kfyXGYJ100).

\section{Disclosure}

The authors report no conflicts of interest in this work.

\section{References}

1. Wu Z, McGoogan JM. Characteristics of and important lessons from the coronavirus disease 2019 (COVID-19) Outbreak in China: summary of a report of 72314 cases from the Chinese center for disease control and prevention. JAMA. 2020;323(13):1239-1242. doi:10.1001/ jama.2020.2648

2. Chen SL, Feng HY, Xu H, et al. Patterns of deterioration in moderate patients with COVID-19 From Jan 2020 to Mar 2020: a Multi-Center, Retrospective Cohort Study in China. Front Med (Lausanne). 2020;7:567296. doi:10.3389/fmed.2020.567296

3. Islam MA, Kundu S, Alam SS, Hossan T, Kamal MA, Hassan R. Prevalence and characteristics of fever in adult and paediatric patients with coronavirus disease 2019 (COVID-19): a systematic review and meta-analysis of 17515 patients. PLoS One. 2021;16(4):e0249788. doi:10.1371/journal.pone. 0249788

4. Hui DSC, Zumla A. Severe acute respiratory syndrome: historical, epidemiologic, and clinical features. Infect Dis Clin North Am. 2019;33(4):869-889. doi:10.1016/j.idc.2019.07.001

5. Badawi A, Ryoo SG. Prevalence of comorbidities in the Middle East respiratory syndrome coronavirus (MERS-CoV): a systematic review and meta-analysis. Int $J$ Infect Dis. 2016;49:129-133. doi:10.1016/j. ijid.2016.06.015 
6. Ws Chew N, Nicholas Ngiam J, Meng Tham S, et al. Fever as a predictor of adverse outcomes in COVID-19. QJM. 2021. doi:10.1093/qjmed/hcab023

7. Tharakan S, Nomoto K, Miyashita S, Ishikawa K. Body temperature correlates with mortality in COVID-19 patients. Crit Care. 2020;24 (1):298. doi:10.1186/s13054-020-03045-8

8. Shu L, Wang X, Li M, et al. Clinical characteristics of moderate COVID-19 patients aggravation in Wuhan Stadium Cabin Hospital: a 571 cases of retrospective cohort study. J Med Virol. 2021;93 (2):1133-1140. doi:10.1002/jmv.26414

9. Wong SH, Lui RN, Sung JJ. Covid-19 and the digestive system. J Gastroenterol Hepatol. 2020;35(5):744-748. doi:10.1111/jgh.15047

10. Han C, Duan C, Zhang S, et al. Digestive Symptoms in COVID-19 patients with mild disease severity: clinical presentation, stool viral RNA testing, and outcomes. Am J Gastroenterol. 2020;115 (6):916-923. doi:10.14309/ajg.0000000000000664

11. Ma C, Cong Y, Zhang H. COVID-19 and the Digestive System. Am J Gastroenterol. 2020;115(7):1003-1006. doi:10.14309/ ajg.0000000000000691

12. Schirmer M, Smeekens SP, Vlamakis H, et al. Linking the human gut microbiome to inflammatory cytokine production capacity. Cell. 2016;167(4):1125-1136 e1128. doi:10.1016/j.cell.2016.10.020

13. Belkaid Y, Hand TW. Role of the microbiota in immunity and inflammation. Cell. 2014;157(1):121-141. doi:10.1016/j.cell.2014.03.011

14. Mahmudpour M, Roozbeh J, Keshavarz M, Farrokhi S, Nabipour I. COVID-19 cytokine storm: the anger of inflammation. Cytokine. 2020;133:155151. doi:10.1016/j.cyto.2020.155151

15. Abbasifard M, Khorramdelazad H. The bio-mission of interleukin- 6 in the pathogenesis of COVID-19: a brief look at potential therapeutic tactics. Life Sci. 2020;257:118097. doi:10.1016/j.1fs.2020.118097

16. Fathi N, Rezaei N. Lymphopenia in COVID-19: therapeutic opportunities. Cell Biol Int. 2020;44(9):1792-1797. doi:10.1002/cbin.11403

17. Moore JB, June CH. Cytokine release syndrome in severe COVID-19. Science. 2020;368(6490):473-474. doi:10.1126/science.abb8925

18. Schirmer M, Kumar V, Netea MG, Xavier RJ. The causes and consequences of variation in human cytokine production in health. Curr Opin Immunol. 2018;54:50-58. doi:10.1016/j.coi.2018.05.012

19. Evans SS, Repasky EA, Fisher DT. Fever and the thermal regulation of immunity: the immune system feels the heat. Nat Rev Immunol. 2015;15(6):335-349. doi:10.1038/nri3843

20. Zuo T, Zhang F, Lui GCY, et al. Alterations in gut microbiota of patients with COVID-19 during time of hospitalization. Gastroenterology. 2020;159(3):944-955 e948. doi:10.1053/j.gastro.2020.05.048

21. Yeoh YK, Zuo T, Lui GC, et al. Gut microbiota composition reflects disease severity and dysfunctional immune responses in patients with COVID-19. Gut. 2021;70(4):698-706. doi:10.1136/gutjnl-2020-323020

22. National Health Commission of the People's Republic of China. Chinese clinical guidance for COVID-19 pneumonia diagnosis and treatment (7th edition). Available from: http://www.nhc.gov.cn/jkj/ s3577/202009/318683cbfaee4191aee29cd774b19d8d/files/ f9ea38ce2c2d4352bf61ab0feada439f.pdf. Accessed May 14, 2021.

23. Di Ciaula A, Stella A, Bonfrate L, Wang DQH, Portincasa P. Gut microbiota between environment and genetic background in familial Mediterranean fever (FMF). Genes (Basel). 2020;11:9. doi:10.3390/ genes 11091041

24. Haak BW, de Jong HK, Kostidis S, et al. Altered patterns of compositional and functional disruption of the gut microbiota in typhoid fever and nontyphoidal febrile illness. Open Forum Infect Dis. 2020;7(7):ofaa251. doi:10.1093/ofid/ofaa251

25. Shehab El-Din EMR, Elgaml A, Ali YM, Hassan R. Inhibition of the classical pathway of complement activation impairs bacterial clearance during Enterococcus faecalis infection. Infect Immun. 2021. doi:10.1128/IAI.00660-20
26. Ran S, Huang J, Liu B, Gu S, Jiang W, Liang J. Enterococcus Faecalis activates NLRP3 inflammasomes leading to increased interleukin-1 beta secretion and pyroptosis of THP-1 macrophages. Microb Pathog. 2021;154:104761. doi:10.1016/j.micpath.2021.104761

27. Nash AK, Auchtung TA, Wong MC, et al. The gut mycobiome of the human microbiome project healthy cohort. Microbiome. 2017;5 (1):153. doi:10.1186/s40168-017-0373-4

28. Candelli M, Nista EC, Nestola M, et al. Saccharomyces cerevisiae-associated diarrhea in an immunocompetent patient with ulcerative colitis. $J$ Clin Gastroenterol. 2003;36(1):39-40. doi:10.1097/00004836-200301000-00012

29. Liu L, Song L, Deng R, et al. Citrobacter freundii activates the NLRP3 inflammasome via T6SS. J Infect Dis. 2020. doi:10.1093/ infdis/jiaa692

30. Sierra Y, Gonzalez-Diaz A, Tubau F, et al. Emergence of multidrug resistance among Haemophilus parainfluenzae from respiratory and urogenital samples in Barcelona, Spain. Eur J Clin Microbiol Infect Dis. 2020;39(4):703-710. doi:10.1007/s10096-019-03774-х

31. Xiaomin S, Yiming L, Yuying Y, Zhangqi S, Yongning W, Shaolin W. Global impact of mcr-1-positive Enterobacteriaceae bacteria on "one health". Crit Rev Microbiol. 2020;46(5):565-577. doi:10.1080/ 1040841X.2020.1812510

32. Sofi MH, Wu Y, Ticer T, et al. A single strain of Bacteroides fragilis protects gut integrity and reduces GVHD. JCI Insight. 2021;6(3). doi:10.1172/jci.insight.136841.

33. Li K, Hao Z, Du J, Gao Y, Yang S, Zhou Y. Bacteroides thetaiotaomicron relieves colon inflammation by activating aryl hydrocarbon receptor and modulating $\mathrm{CD} 4(+) \mathrm{T}$ cell homeostasis. Int Immunopharmacol. 2021;90:107183. doi:10.1016/j. intimp.2020.107183

34. Rodriguez-Castano GP, Dorris MR, Liu X, Bolling BW, AcostaGonzalez A, Rey FE. Bacteroides thetaiotaomicron starch utilization promotes quercetin degradation and butyrate production by eubacterium ramulus. Front Microbiol. 2019;10:1145. doi:10.3389/ fmicb.2019.01145

35. Ulsemer P, Toutounian K, Schmidt J, Karsten U, Goletz S. Preliminary safety evaluation of a new Bacteroides xylanisolvens isolate. Appl Environ Microbiol. 2012;78(2):528-535. doi:10.1128/ AEM.06641-11

36. Wang Y, Shao S, Guo C, Zhang S, Li M, Ding K. The homogenous polysaccharide SY01-23 purified from leaf of Morus alba L. has bioactivity on human gut Bacteroides ovatus and Bacteroides cellulosilyticus. Int $J$ Biol Macromol. 2020;158:698-707. doi:10.1016/j.ijbiomac.2020.05.009

37. Ricci C, Ortore MG, Vilasi S, et al. Stability and disassembly properties of human naive Hsp60 and bacterial GroEL chaperonins. Biophys Chem. 2016;208:68-75. doi:10.1016/j.bpc.2015.07.006

38. Elfaitouri A, Herrmann B, Bolin-Wiener A, et al. Epitopes of microbial and human heat shock protein 60 and their recognition in myalgic encephalomyelitis. PLoS One. 2013;8(11):e81155. doi:10.1371/ journal.pone. 0081155

39. Kol A, Bourcier T, Lichtman AH, Libby P. Chlamydial and human heat shock protein 60 s activate human vascular endothelium, smooth muscle cells, and macrophages. J Clin Invest. 1999;103(4):571-577. doi:10.1172/JCI5310

40. Eskilsson A, Mirrasekhian E, Dufour S, Schwaninger M, Engblom D, Blomqvist A. Immune-induced fever is mediated by IL- 6 receptors on brain endothelial cells coupled to STAT3-dependent induction of brain endothelial prostaglandin synthesis. J Neurosci. 2014;34 (48):15957-15961. doi:10.1523/JNEUROSCI.3520-14.2014

41. Choron RL, Butts CA, Bargoud C, et al. Fever in the ICU: a Predictor of Mortality in Mechanically Ventilated COVID-19 Patients. $J$ Intensive Care Med. 2021;36(4):484-493. doi:10.1177/ 0885066620979622 


\section{Publish your work in this journal}

The Journal of Inflammation Research is an international, peerreviewed open-access journal that welcomes laboratory and clinical findings on the molecular basis, cell biology and pharmacology of inflammation including original research, reviews, symposium reports, hypothesis formation and commentaries on: acute/chronic inflammation; mediators of inflammation; cellular processes; molecular mechanisms; pharmacology and novel anti-inflammatory drugs; clinical conditions involving inflammation. The manuscript management system is completely online and includes a very quick and fair peerreview system. Visit http://www.dovepress.com/testimonials.php to read real quotes from published authors.

Submit your manuscript here: https://www.dovepress.com/journal-of-inflammation-research-journal 